\title{
Electromagnetic Oscillations in a Driven Nonlinear Resonator: A New Description of Complex Nonlinear Dynamics
}

\author{
E. Yu. Petrov and A. V. Kudrin* \\ Department of Radiophysics, University of Nizhny Novgorod, \\ 23 Gagarin Ave., Nizhny Novgorod 603950, Russia
}

\begin{abstract}
Many intriguing properties of driven nonlinear resonators, including the appearance of chaos, are very important for understanding the universal features of nonlinear dynamical systems and can have great practical significance. We consider a cylindrical cavity resonator driven by an alternating voltage and filled with a nonlinear nondispersive medium. It is assumed that the medium lacks a center of inversion and the dependence of the electric displacement on the electric field can be approximated by an exponential function. We show that the Maxwell equations are integrated exactly in this case and the field components in the cavity are represented in terms of implicit functions of special form. The driven electromagnetic oscillations in the cavity are found to display very interesting temporal behavior and their Fourier spectra contain singular continuous components. To the best of our knowledge, this is the first demonstration of the existence of a singular continuous (fractal) spectrum in an exactly integrable system.
\end{abstract}

PACS numbers: 05.45.-a

Nonlinear resonators are simple and convenient models of physical systems and have proven to be a valuable means to investigate the universal features of nonlinear dynamics. In view of this, such resonators have been the subject of intense theoretical and experimental studies in the past decades [1-4]. A variety of the existing electronic devices and materials with nonlinear electromagnetic properties makes it possible to create electrical resonators with different types of nonlinearity. From a practical viewpoint, it is important that when electromagnetic oscillations in a bounded volume are excited at frequencies close to resonant ones, oscillation amplitudes that are sufficient for manifestation of various nonlinear phenomena can be reached even if a driving source is comparatively weak. The well-known example of such phenomena is generation of harmonics of a drive frequency, which enables realization of efficient frequency multipliers on the basis of high-Q resonators. In addition, fairly complex, e.g., chaotic, oscillations can be excited in nonlinear resonators [1 3$]$. Studying chaotic regimes of resonators is of great interest for developing controllable sources of noise-like signals. In more recent works, a new type of complex nonlinear dynamics, intermediate between almost periodic and random, has been discovered [5-7]. Such dynamics, which is associated with a singular continuous spectrum, appears typically in driven nonlinear systems and has received much attention [814], both for fundamental reasons and because of its interdisciplinary relevance [7].

A complete description of the complex dynamics of distributed nonlinear systems is fairly difficult to achieve. This is explained by an infinite number of degrees of freedom and the presence of several controlling parameters in such systems. Because of this, most theoretical works on the subject discuss nonlinear resonators as lumped systems or merely as a collection of coupled oscillators or modes. Within the framework of such a simplified approach, the problem of oscillations in a nonlinear resonator is reduced to solving a system of ODEs. Although such an approach is justified in many cases, it is clear that electromagnetic systems should generally be described by the Maxwell equations.

In this work, the problem of a nonlinear electrical resonator is considered using a full set of the Maxwell equations. In what follows, we apply the method for constructing exact axisymmetric solutions of the Maxwell equations in a nonlinear nondispersive medium, which has been developed in our recent works [15, 16], to the driven oscillations in a bounded volume.

Consider electromagnetic fields in a cylindrical cavity of radius $a$ and height $L$. We assume that the $z$ axis of a cylindrical coordinate system $(r, \phi, z)$ is aligned with the cavity axis and limit ourselves to consideration of axisymmetric field oscillations in which only the $E_{z}$ and $H_{\phi}$ components are nonzero. We will also assume that the cavity is filled with a nonlinear nondispersive medium in which the longitudinal component of the electric displacement can be represented as $D_{z}=D_{0}+\alpha^{-1} \epsilon_{0} \varepsilon_{1}\left[\exp \left(\alpha E_{z}\right)-1\right]$, where $D_{0}, \varepsilon_{1}$, and $\alpha$ are certain constants. The possibility of using such a model of nonlinearity for media lacking a center of inversion is discussed in detail in [15]. As is shown in [15], this model, with appropriately chosen $D_{0}, \varepsilon_{1}$, and $\alpha$, correctly describes dielectric properties of such media in the case of moderately small electric fields. Then the Maxwell equations read

$$
\partial_{r} H+r^{-1} H=\varepsilon(E) \partial_{t} E, \quad \partial_{r} E=\mu_{0} \partial_{t} H,
$$

where $E \equiv E_{z}(r, t), H \equiv H_{\phi}(r, t)$, and

$$
\varepsilon(E) \equiv d D_{z} / d E=\epsilon_{0} \varepsilon_{1} \exp (\alpha E) .
$$

The exact solution of system (1) can be written in im- 
plicit form as [15]

$$
\begin{aligned}
\tilde{E} & =A^{-1} \mathcal{E}\left(\rho e^{\tilde{\alpha} \tilde{E} / 2}, \tau+\tilde{\alpha} \rho \tilde{H} / 2\right), \\
\tilde{H} & =e^{\tilde{\alpha} \tilde{E} / 2} A^{-1} \mathcal{H}\left(\rho e^{\tilde{\alpha} \tilde{E} / 2}, \tau+\tilde{\alpha} \rho \tilde{H} / 2\right) .
\end{aligned}
$$

Hereafter, $A$ is a constant amplitude factor related to the field source, $\tilde{E}=E / A, \tilde{H}=Z_{0} H /\left(A \varepsilon_{1}^{1 / 2}\right), \rho=r / a, \tau=$ $t\left(\epsilon_{0} \varepsilon_{1} \mu_{0}\right)^{-1 / 2} / a$, and $\tilde{\alpha}=\alpha A$, where $Z_{0}=\left(\mu_{0} / \epsilon_{0}\right)^{1 / 2}$. The functions $\mathcal{E}$ and $\mathcal{H}$ describe the electromagnetic field in a linear medium and satisfy the equations

$$
\partial_{\rho}^{2} \mathcal{E}+\rho^{-1} \partial_{\rho} \mathcal{E}=\partial_{\tau}^{2} \mathcal{E}
$$

and $\partial_{\rho} \mathcal{E}=\partial_{\tau} \mathcal{H}$.

Let us state the following initial and boundary conditions for linear wave equation (4):

$$
\begin{aligned}
& \mathcal{E}(\rho, 0)=0, \quad \partial_{\tau} \mathcal{E}(\rho, 0)=0, \quad 0 \leq \rho<1, \\
& \mathcal{E}(1, \tau)=A\left(\sin \Omega_{1} \tau+0.5 \sin \Omega_{2} \tau\right), \quad 0 \leq \tau<\infty,
\end{aligned}
$$

where $\Omega_{1,2}$ are normalized constant frequencies such that $\Omega_{1,2}=\lambda_{1,2} a\left(\epsilon_{0} \varepsilon_{1} \mu_{0}\right)^{1 / 2}$ (i.e., $\left.\Omega_{1,2} \tau=\lambda_{1,2} t\right)$. The frequencies $\Omega_{1}$ and $\Omega_{2}$ are related as $\Omega_{1}=\sigma \Omega_{2}$, where $\sigma=(\sqrt{5}-1) / 2$ is the golden mean. The boundary value problem defined by Eqs. (4) -(6) describes the driven electromagnetic oscillations in a cylindrical cavity specified by the relations $\rho=r / a \leq 1$ and $0 \leq z \leq L$, which is filled with a linear medium having the permittivity $\varepsilon=\epsilon_{0} \varepsilon_{1}=$ const $(\alpha=0)$. The linear oscillations are thus driven by an electric field (6) which can be produced by two coaxial metal rings of radius $a$ that are separated by distance $L$ if an almost periodic voltage $V=\mathcal{E}(1, \tau) L=A L\left(\sin \lambda_{1} t+0.5 \sin \lambda_{2} t\right)$ is applied between them. The solution to the linear boundary value problem specified by Eqs. (4) -(6) can be found in a standard way [17]. As a result, the functions $\mathcal{E}$ and $\mathcal{H}$ are written as

$$
\begin{aligned}
& \mathcal{E}(\rho, \tau)=\sum_{j=1}^{2} B_{j} \frac{J_{0}\left(\Omega_{j} \rho\right)}{J_{0}\left(\Omega_{j}\right)} \sin \Omega_{j} \tau+\sum_{n=1}^{\infty} C_{n} J_{0}\left(\kappa_{n} \rho\right) \sin \kappa_{n} \tau, \\
& \mathcal{H}(\rho, \tau)=\sum_{j=1}^{2} B_{j} \frac{J_{1}\left(\Omega_{j} \rho\right)}{J_{0}\left(\Omega_{j}\right)} \cos \Omega_{j} \tau+\sum_{n=1}^{\infty} C_{n} J_{1}\left(\kappa_{n} \rho\right) \cos \kappa_{n} \tau
\end{aligned}
$$

where $B_{j}=A / j, C_{n}=2 A \sum_{j=1}^{2} \Omega_{j}\left[j\left(\Omega_{j}^{2}-\kappa_{n}^{2}\right) J_{1}\left(\kappa_{n}\right)\right]^{-1}$, $J_{m}$ is the Bessel function of the first kind of order $m, \kappa_{n}$ is the $n$th root of the equation $J_{0}(\kappa)=0$, and $\Omega_{1.2} \neq \kappa_{n}$. We denote eigenfrequencies of the $E_{0 n 0}$ modes [18] of a linear resonator as $\omega_{n}$. Hence, $\omega_{n}=\kappa_{n}\left(\epsilon_{0} \mu_{0}\right)^{-1 / 2} a^{-1}$ and $\kappa_{n} \tau=\omega_{n} t$.

Substituting functions (7) into formulas (3), we obtain an exact solution to system (11) in implicit form. Thus, the field components $E$ and $H$ in a cylindrical cavity filled with a nonlinear medium for which $\varepsilon(E)$ is written in the form of Eq. (2) are found from the solution of a set of transcendental equations (3) in which $\mathcal{E}$ and $\mathcal{H}$, defined by relationships (7), are almost periodic functions of $\tau$. In the nonlinear case, the fields $E$ and $H$, which are determined by Eqs. (3) and (7), satisfy the same initial conditions as in Eq. (5):

$$
E(\rho, 0)=0, \quad \partial_{\tau} E(\rho, 0)=0, \quad 0 \leq \rho<1 .
$$

However, the electric field oscillations on the side wall of the nonlinear resonator $(\rho=1)$ in the found solution do not obey Eq. (6). Putting $\rho=1$ in formulas (3), we have

$$
\begin{aligned}
& \tilde{E}=A^{-1} \mathcal{E}\left(e^{\tilde{\alpha} \tilde{E} / 2}, \tau+\tilde{\alpha} \tilde{H} / 2\right), \\
& \tilde{H}=e^{\tilde{\alpha} \tilde{E} / 2} A^{-1} \mathcal{H}\left(e^{\tilde{\alpha} \tilde{E} / 2}, \tau+\tilde{\alpha} \tilde{H} / 2\right) .
\end{aligned}
$$

The dependence $\tilde{E}(1, \tau)$ determined by relationships (9) can be regarded as a drive signal in the boundary value problem given by Eqs. (11), (8), and (9) for a nonlinear $(\alpha \neq 0)$ resonator.

Thus, formulas (3), with $\mathcal{E}$ and $\mathcal{H}$ given by relationships (7), yield an exact solution of the nonlinear boundary value problem for system (11) under conditions (8) and (9), and describe driven electromagnetic oscillations in a cylindrical cavity resonator filled with a nonlinear medium. A typical example of such a medium can be a ferroelectric crystal. Note that ferroelectric resonators are known to be of great interest for many promising applications [4].

Observe that the oscillations on the axis $\rho=0$ of the nonlinear resonator in the obtained exact solution coincide with the oscillations for $\rho=0$ in the "seeding" linear problem. It follows from Eqs. (3) and (7) that for $\rho=0$, $E(0, \tau) \equiv \mathcal{E}(0, \tau)=\sum_{j=1}^{2} B_{j} \sin \Omega_{j} \tau+\sum_{n=1}^{\infty} C_{n} \sin \kappa_{n} \tau$ and $H(0, \tau) \equiv \mathcal{H}(0, \tau)=0$. Thus, the electric field oscillations on the axis $\rho=0$ are described by an almost periodic function of $\tau$ and have the discrete spectrum [19].

For $\rho \neq 0$, the exact solution expressed in terms of implicit functions is very complicated and can be studied only numerically. It turns out that for $\rho \neq 0$, the field oscillations described by this solution may have singular continuous (fractal) spectrum.

To confirm the above assertion, we turn to results of calculations of the quantities $\tilde{E}$ and $\tilde{H}$ determined by Eqs. (3) and (77). In what follows, the main attention will be focused on analyzing the obtained solutions in the case where the drive frequency $\lambda_{2}$ relates to the fundamental eigenfrequency $\omega_{1}$ of a linear resonator as $\lambda_{2} / \omega_{1}=\Omega_{2} / \kappa_{1}=\sigma$ (note that the identity $\Omega_{1}+\Omega_{2}=\kappa_{1}$ is valid in this case). In our calculations, we retain 100 terms of the series over $n$ in formulas (7). It should be noted that the employed theoretical model of a nondispersive medium does not allow one to indefinitely increase the nonlinearity parameter $\tilde{\alpha}$ in solutions (3). For large absolute values of $\tilde{\alpha}$, implicit functions $\tilde{E}(\rho, \tau)$ and $\tilde{H}(\rho, \tau)$ determined by Eqs. (3) and (7) become ambiguous, and solutions (3) obtained without allowance for dispersion will be inapplicable [15]. For fixed $\tilde{\alpha}$ and $\Omega_{1,2}$, 
the ambiguity points appear first in the time dependences $\tilde{E}(1, \tau)$ and $\tilde{H}(1, \tau)$ (for $\rho=1$ ) since the nonlinear effects are accumulating with increasing $\rho$ [15]. Therefore, as a first step in practical calculations, one should study the functions $\tilde{E}(1, \tau)$ and $\tilde{H}(1, \tau)$. If these functions are unambiguous and continuous, then $\tilde{E}$ and $\tilde{H}$ possess the same properties for $0 \leq \rho<1$. In all the computations, we use the maximum possible value $\tilde{\alpha}=0.32$ for chosen $\Omega_{1,2}$.

Now consider the field oscillations on the wall $\rho=1$ of the nonlinear resonator. The dependences $\tilde{E}(1, \tau)$ and $\tilde{H}(1, \tau)$ determined by relationships (9) are shown in Fig. 1(a) by the red and blue lines, respectively. For comparison, the solid and dashed black lines in Fig.1 (a) show the functions $\mathcal{E}$ and $\mathcal{H}$, respectively. It is seen in Fig. 1 that the functions $\tilde{E}$ and $\tilde{H}$ demonstrate fairly complex behavior and essentially differ from the corresponding quantities $\mathcal{E} / A$ and $\mathcal{H} / A$ in the linear regime $(\alpha=0)$ by the presence of small amplitude spikes. Figures $1(\mathrm{~b})$ and 1 (c) show the frequency spectra of $\tilde{E}(1, \tau)$ and $\tilde{H}(1, \tau)\left(2^{16}\right.$ points were taken for the FFT with a sampling rate of 0.05$)$. The FFT plots exhibit numerous spectral components that can be attributed to the harmonics of $\Omega_{1,2}$ and $\kappa_{n}$, as well as various combination frequencies.

To reveal nontrivial spectral properties of the oscillations considered, we use the singular-continuous spectrum analysis [6, 9 [1], 13]. We define the partial Fourier sums

$$
S_{E}(\Omega, T)=\sum_{m=1}^{T} \tilde{E}_{m} e^{i \Omega \tau_{m}}, \quad S_{H}(\Omega, T)=\sum_{m=1}^{T} \tilde{H}_{m} e^{i \Omega \tau_{m}},
$$

where $\left\{\tilde{E}_{m}\right\}$ and $\left\{\tilde{H}_{m}\right\}$ are the time series of the variables $\tilde{E}$ and $\tilde{H}: \tilde{E}_{m}=\tilde{E}\left(\tau_{m}\right)$ and $\tilde{H}_{m}=\tilde{H}\left(\tau_{m}\right)$. We take $\tau_{1}=$ 100 and $\tau_{m+1}-\tau_{m}=0.02$, although all the forthcoming results are valid when changing the initial point and the sampling rate. The Fourier transforms scale with $T$ as

$$
\left|S_{E}(\Omega, T)\right|^{2} \sim T^{\beta}, \quad\left|S_{H}(\Omega, T)\right|^{2} \sim T^{\gamma},
$$

where $\beta=\beta(\Omega)$ and $\gamma=\gamma(\Omega)$ are scaling exponents [6, 9, 11]. The evolution of $S_{E}$ and $S_{H}$ with $T$ can be represented by paths in the complex planes $\left(\operatorname{Re} S_{E}\right.$, $\left.\operatorname{Im} S_{E}\right)$ and $\left(\operatorname{Re} S_{H}, \operatorname{Im} S_{H}\right)$, respectively. It is known [9] that for $\beta=\gamma=2$, the frequency $\Omega$ belongs to the countable set of discrete spectral components of an almost periodic oscillation and there exist persistent motions (drifts) of $S_{E}$ and $S_{H}$ in the corresponding complex planes. A singular-continuous spectral component appears if (i) $\beta \neq 1,2$ and/or $\gamma \neq 1,2$ and (ii) the path in the complex plane is fractal [6, 9 11, 13]. A singular continuous spectrum is known to be a Cantor set [7, 14].

We have found that at some frequencies, the spectrum has the scaling exponents $\beta=\gamma=2$. Figures 2(a) and 2(b) show $\left|S_{E}\right|^{2}$ and $\left|S_{H}\right|^{2}$ as functions of $\log _{10} T$ for $\rho=1$
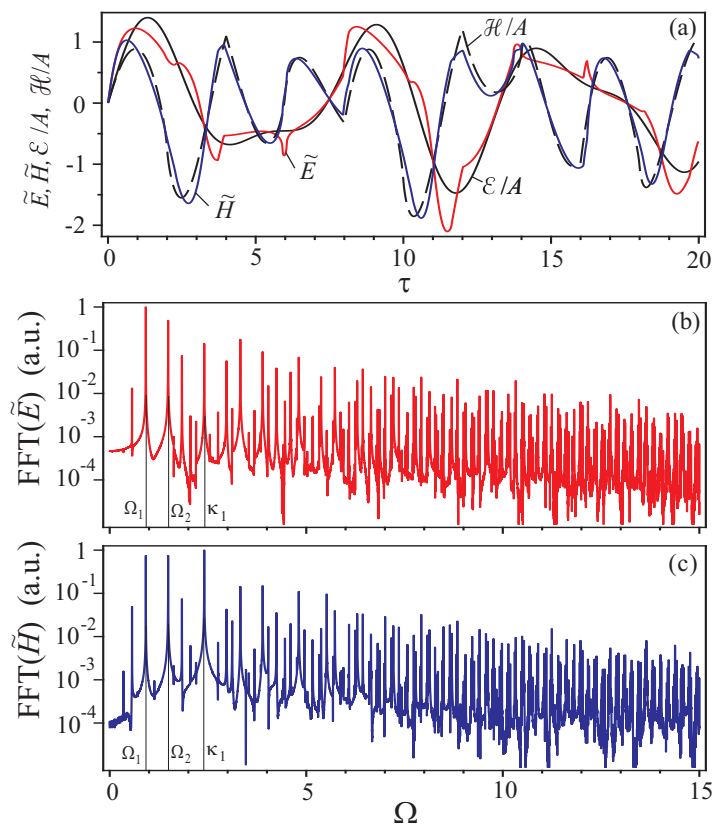

FIG. 1. (color online) (a) Oscillograms of the electric $(\tilde{E})$ and magnetic $(\tilde{H})$ fields on the wall $\rho=1$ of a nonlinear resonator (red and blue lines, respectively), calculated by formulas (9), and the corresponding quantities $\mathcal{E} / A$ and $\mathcal{H} / A$ in the linear regime (solid and dashed black lines, respectively). The fast Fourier transforms of (b) $\tilde{E}$ and (c) $\tilde{H}$.

at one of such frequencies, namely, $\Omega=\kappa_{3}=8.65 \ldots$. The corresponding paths in the complex planes are presented in Figs. 2(c) and 2(d). Thus, in this case, we deal with a discrete component of the spectrum. However, at the combination frequency $\Omega_{7,3,4}=32.96 \ldots$ (hereafter, $\left.\Omega_{l, m, n}=l \Omega_{1}+m \Omega_{2}+n \kappa_{2}\right)$, we have $\beta \approx 1.7$ and $\gamma \approx 1.62$ [see Figs. 2(a) and 2(b)], and the behavior of the dependences $\left|S_{E}\right|^{2}$ and $\left|S_{H}\right|^{2}$ in this case is typical of a singular continuous component [9, 11, 13]. The corresponding paths in Figs. 2(e) and 2(f) exhibit fractal structures. These results strongly suggest that the considered spectrum of electromagnetic oscillations is not purely discrete and contains singular continuous components. For $\rho=1$, we have also found such components at, e.g., the combination frequencies $\Omega_{3,3,4}(\beta \approx 1.57$ and $\gamma=0), \Omega_{3,4,4}$ $(\beta \approx 1.6$ and $\gamma=0), \Omega_{6,3,4}(\beta \approx 1.53$ and $\gamma \approx 1.4)$, $\Omega_{3,-1,4}(\beta \approx 1.1$ and $\gamma \approx 1.55), \Omega_{6,5,4}(\beta \approx 1.52$ and $\gamma \approx 1.8)$, and $\Omega_{5,6,4}(\beta \approx 1.4$ and $\gamma \approx 1.2)$. For many frequencies, a power-law growth of the spectrum is observed with the exponents $\beta$ and $\gamma$ which differ from 2 only slightly.

We now pass to consideration of some spectral features of oscillations inside a nonlinear resonator for $\rho=0.5$. Here, the components of the singular continuous spectrum appear at higher frequencies than for $\rho=1$. The values of $\beta$ and $\gamma$ for $\rho=0.5$ turn out to be smaller than for $\rho=1$ at the same frequency. For example, at $\Omega=\Omega_{6,5,4}=35.02 \ldots$, we have $\beta \approx 1.52$ and $\gamma \approx 1.8$ 

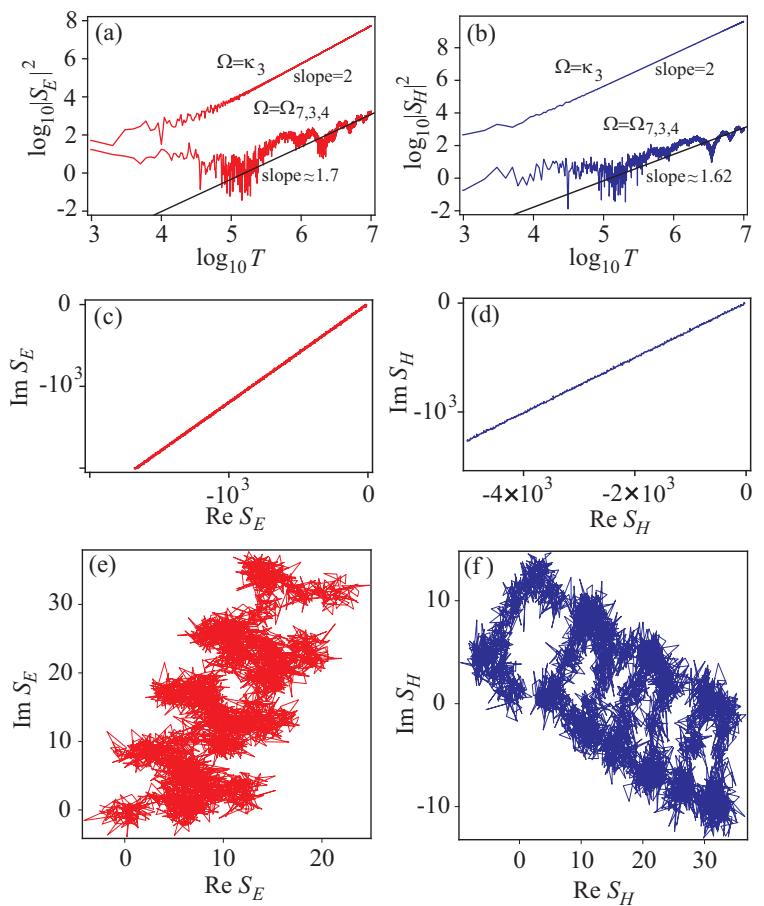

FIG. 2. (color online) Singular-continuous spectrum analysis of the time series $\left\{\tilde{E}_{m}\right\}$ and $\left\{\tilde{H}_{m}\right\}$ for $\rho=1$. (a) $\left|S_{E}\right|^{2}$ and (b) $\left|S_{H}\right|^{2}$ as functions of $\log _{10} T$ at $\Omega=\kappa_{3}$ and $\Omega=\Omega_{7,3,4}$. The paths of (c) $S_{E}$ and (d) $S_{H}$ in the complex planes (Re $S_{E}$, $\left.\operatorname{Im} S_{E}\right)$ and $\left(\operatorname{Re} S_{H}, \operatorname{Im} S_{H}\right)$, respectively, at $\Omega=\kappa_{3}$. The paths of (e) $S_{E}$ and (f) $S_{H}$ in the same planes at $\Omega=\Omega_{7,3,4}$.

for $\rho=1$, and $\beta \approx 1.05$ and $\gamma \approx 1.05$ for $\rho=0.5$ [see Figs. 3(a) and 3(b)]. The corresponding curves in the complex planes $\left(\operatorname{Re} S_{E}, \operatorname{Im} S_{E}\right)$ and $\left(\operatorname{Re} S_{H}, \operatorname{Im} S_{H}\right)$, which are presented in Figs. 3(c) and 3(d), display fractal behavior.

Thus, our analysis shows that the Fourier spectrum of the electromagnetic oscillations in the cavity is a mixture of discrete and singular continuous components. Similar phenomena have been reported in the literature and discussed in, e.g., [9, 14] as applied to the dynamics described by forced maps and symbolic sequences. In our case, calculations of the FFT and the autocorrelation function $(\mathrm{ACF})$ do not allow one to adequately investigate the spectral properties of the considered oscillations. Because of the weakness of the contribution from the singular continuous part, the FFT and the ACF are similar to those of almost periodic motion. Scaling is a powerful method to detect the presence of a singular continuous spectrum. However, it is not clear how this component can be separated from the discrete spectrum.

The existence of regimes with singular continuous (fractal) spectra in dissipative dynamical systems described by discrete maps or ODEs is well known [8 11]. Such regimes corresponding to strange nonchaotic attractors are realized on sets of positive measure in the parameter spaces of dissipative dynamical systems and are typ-
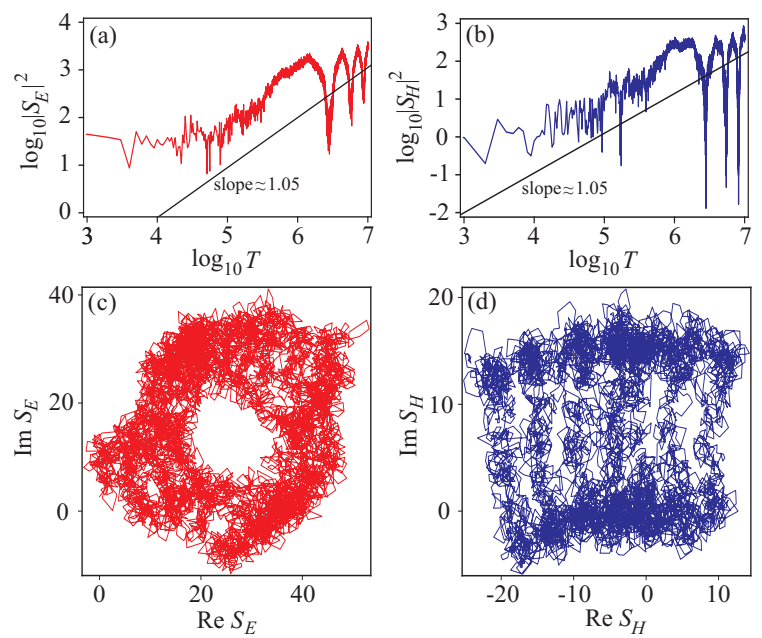

FIG. 3. (color online) Singular-continuous spectrum analysis of the time series $\left\{\tilde{E}_{m}\right\}$ and $\left\{\tilde{H}_{m}\right\}$ for $\rho=0.5$. (a) $\left|S_{E}\right|^{2}$ and (b) $\left|S_{H}\right|^{2}$ as functions of $\log _{10} T$ at $\Omega=\Omega_{6,5,4}$. The corresponding paths of (c) $S_{E}$ and (d) $S_{H}$ in the complex planes.

ical of the intermediate region between almost periodic and random motions. Our study demonstrates that the nonlinear dynamics with a singular continuous spectrum can occur in an exactly integrable distributed nondissipative system. We have found that the implicit functions given by Eqs. (3) and (7), which are exact solutions of system (1), are not almost periodic in $\tau$ and their Fourier spectra contain singular continuous components. Studying these functions is of great interest for physics and mathematics. We have shown that such functions can be finite-amplitude single-valued solutions of the Maxwell equations and, hence, describe actually existing electromagnetic oscillations. Thus, Eqs. (3) and (7) provide a new description of complex nonlinear dynamics.

This work was supported by the Russian Foundation for Basic Research (Project No. 12-02-00904-a) and the Ministry of Education and Science of the Russian Federation (Contract Nos. P313 and 11.G34.31.0048). A. V. K. acknowledges partial support from the Greek Ministry of Education under the project THALIS (RF-EIGENSDR).

* kud@rf.unn.ru

[1] P. S. Linsay, Phys. Rev. Lett. 47, 1349 (1981)

[2] R. Van Buskirk and C. Jeffries, Phys. Rev. A 31, 3332 (1985)

[3] J. H. Baxter, M. F. Bocko, and D. H. Douglass, Phys. Rev. A 41, 619 (1990)

[4] I. V. Ivanov, Sov. Phys. Usp. 23, 869 (1980)

[5] C. Grebogi, E. Ott, S. Pelikan, and J. A. Yorke, Physica D 13, $261(1984)$

[6] S. Aubry, C. Codreche, and J. M. Luck, J. Stat. Phys. 
51, 1033 (1988)

[7] J. M. Luck, Phys. Rev. B 39, 5834 (1989)

[8] M. Ding, C. Grebogi, and E. Ott, Phys. Rev. A 39, 2593 (1989)

[9] A. S. Pikovsky and U. Feudel, J. Phys. A: Math. Gen. 27, 5209 (1994)

[10] A. S. Pikovsky, M. A. Zaks, U. Feudel, and J. Kurths, Phys. Rev. E 52, 285 (1995)

[11] T. Yalçinkaya and Y.-C. Lai, Phys. Rev. E 56, 1623 (1997)

[12] B. P. Bezruchko, S. P. Kuznetsov, and Y. P. Seleznev, Phys. Rev. E 62, 7828 (2000)

[13] M. Agrawal, A. Prasad, and R. Ramaswamy, Phys. Rev.
E 81, 026202 (2010)

[14] M. A. Zaks, Fractal Fourier Spectra in Dynamical Systems (Universität Potsdam, Potsdam, 2001)

[15] E. Yu. Petrov and A. V. Kudrin, Phys. Rev. Lett. 104, 190404 (2010)

[16] V. A. Es'kin, A. V. Kudrin, and E. Yu. Petrov, Phys. Rev. E 83, 067602 (2011)

[17] R. Courant and D. Hilbert, Methods of Mathematical Physics (Wiley, New York, 1966)

[18] J. D. Jackson, Classical Electrodynamics (Wiley, New York, 1998)

[19] H. Bohr, Almost Periodic Functions (Chelsea, New York, 1947) 